\title{
Single plus one port robotic radical prostatectomy (SPORP); Initial experience
}

\author{
Volkan Tugcu, Abdulmuttalip Simsek, Ismail Evren, Kamil Gokhan Seker, Ramazan Kocakaya, \\ Bugra Dogukan Torer, Arda Atar, Ali Ihsan Tasci \\ Department of Urology, Bakirkoy Dr. Sadi Konuk Training and Research Hospital, Istanbul, Turkey.
}

\begin{abstract}
Summary Objective: This article reports on patients with early stage prostate cancer treated with single plus one port robotic radical prostatectomy (SPORP).

Materials and methods: Since January 2014, we performed SPORP in 8 patients with localized prostate cancer. Age of patients, clinical stage, operation time, intraoperative and postoperative complications, blood loss, histopathological evaluation, postoperative continence, serum level of PSA were evaluated.

Results: Mean age of the 8 patients was 59.85 years. All operations were completed without conversion to standard robotic procedure or open surgery. No intra operative complications occurred. Mean operating time was 143 minutes; prostate excision 123 minutes and urethrovesical anastomosis 20 minutes. Mean blood loss was $45 \mathrm{ml}$. Preoperative Gleason scores were $(3+4)$ in one patient and $(3+3)$ in 7 patients.

Postoperative Gleason scores were $(3+4)$ in 2 patients, and $(3+3)$ in 6 patients. All these 8 cases were in T1c clinical stage. Early postoperative complications were drain leakage $(n=1)$, atelectasis $(n=1)$, wound infection $(n=1)$ and fever $(n=1)$. There was no positive surgical margin. The serum level of PSA was less than $0.2 \mathrm{ng} / \mathrm{ml}$ and no other complications happened during the 4 to 12 months follow-up period. Postoperative continence and cosmetic results were excellent. Conclusions: It is relatively easy for urologists who are skilled in traditional laparoscopic and robotic surgeries to master SPORP. However long-term outcomes of this surgery need further investigations.
\end{abstract}

KEY WORDS: Single plus one port robotic radical prostatectomy (SPORP); Prostate cancer; Initial experience; Radical prostatectomy.

Submitted 3 June 2017; Accepted 7 July 2017

\section{INTRODUCTION}

In recent years, laparoendoscopic single-site surgery (LESS) becomes the latest point of minimal invasive surgery. Reducing the number of ports minimizes the complications. Conventional LESS is not easy to learn and perform. In this point $\mathrm{da}$ - Vinci enhances the intracorporeal maneuvering and makes the procedure easier (1-3).

Radical prostatectomy is the first treatment of choice for localized prostate cancer. Robotic radical prostatectomy is improved to reduce the invasiveness and to facilitate the difficulty of open procedure; in this point single port radical prostatectomy is the latest technique $(4,5)$.
The aim of this procedure is to minimize complications associated with number of ports. The present study was designed to define the single plus one port robotic radical prostatectomy (SPORP) technique, and to demonstrate its feasibility and safety.

During the past few years, the detection of prostate cancer and the number of operations are dramatically increased with usage of prostate specific antigen (PSA). Open surgery, laparoscopic surgery and robot-assisted laparoscopic surgery have the same results for trifecta at early stage cancer (5-7). However, urologists throughout the world are searching for more minimally invasive technique. We reported 8 robotic radical prostatectomy performed with single-site port $(8.5 \mathrm{~mm})$ plus one $8 \mathrm{~mm}$ port.

\section{MATERIALS AND METHODS}

\section{Patients}

Since January 2014 we performed SPORP in 8 patients with prostate cancer. Patients with localized prostate cancer were included in the study, and patients with a very high body mass index (BMI) (> $\left.35 \mathrm{~kg} / \mathrm{m}^{2}\right)$ were excluded from the study. All cases were verified as prostate cancer preoperatively by trans-rectal ultrasound guided 10-core biopsy. No pharmacotherapy or radiotherapy was administered preoperatively.

\section{Surgical technique}

Under general anesthesia, patients were placed in Trendelenburg position. Access was gained using open Hasson technique with $3 \mathrm{~cm}$ umbilical longitudinal incision. Rectus fascia was incised and single-site port was inserted transperitoneally. The pneumo-peritoneum was maintained at 12-14 mmHg pressure. $10 \mathrm{~mm}$ assistant port was inserted edge of single- site port without incising the fascia (Figure 1).

Another $8 \mathrm{~mm}$ trocar was placed under direct vision at the McBurney point to ease intra corporeal suturing and drainage extraction (Figure 2).

The procedures were performed technically same as conventional robotic radical prostatectomy with flexible instruments inserted through single-site port and one standard robotic instrument inserted through extra 8 $\mathrm{mm}$ port.

Both deferens vasa were identified and dissected. The sem- 


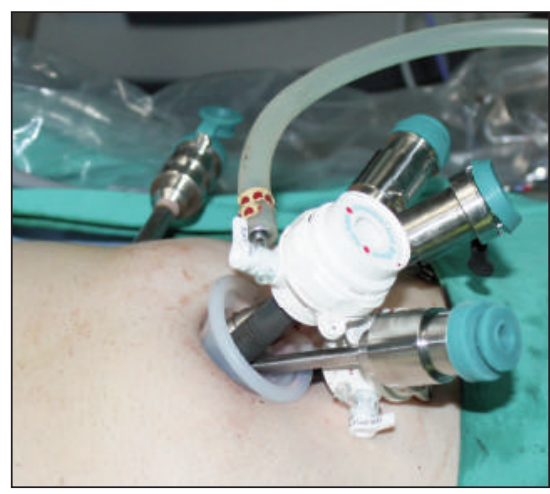

Figure 1.

Port configuration for a SPORP and $10 \mathrm{~mm}$ assistant port.

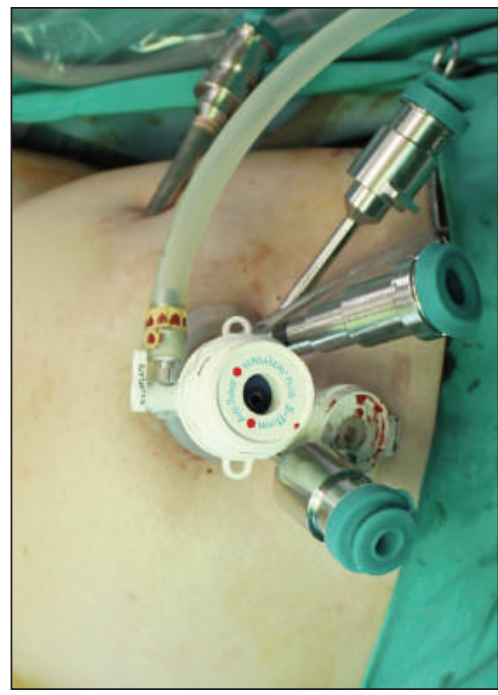

inal vesicles were dissected inferiorly. The dorsal vein complex was over sewed by using a 2-0-polyglycolic acid absorbable suture. The bladder neck was dissected between the bladder neck and prostate by using monopolar scissors and then the urethra was incised. The posterior aspect of bladder neck was dissected and deferens vasa and seminal vesicles were freed bilaterally. The neurovascular bundle was dissected and freed from prostate base by using Hem-o-lock.

The dorsal vein complex and urethra at the prostate apex were incised. The prostate was released from the Denonvillier fascia. After bleeding control, anastomosis was performed with two fixed 3-0 barbed polyglyconate sutures V-LOC (Covidien). Foley catheter was filled with 20 $\mathrm{ml}$ sterile water and the watertight anastomosis was verified by filling bladder with $150 \mathrm{ml}$ saline. The prostate specimen was put into organ bag retrieved through the periumbilical incision. A soft drain was placed through the extra $8 \mathrm{~mm}$ port (Figure 3). Foley catheter was removed 7 days later.

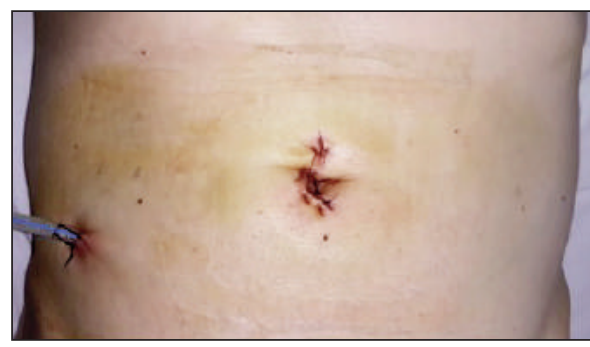

\section{RESULTS}

Since January 2014 we performed SPORP in 8 patients with localized prostate cancer. The mean age was 59.85 years (range 49-71). The mean level of PSA was 6.91 ng/mL (range 5.78-8.81). Preoperative Gleason scores were $(3+4)$ in one patient and $(3+3)$ in 7 patients. All operations were completed successfully without conversion to standard robotic procedure or open surgery. No intra operative complications occurred. The mean operating time was 143 minutes (range 100-180 minutes): prostate excision time was 123 minutes (range 86-156 minutes) and urethrovesical anastomosis time was 20 minutes (range 14-24 minutes). The mean blood loss was $45 \mathrm{ml}$ (range $30-55 \mathrm{ml}$ ).

All patients were in Tlc clinical stage. Postoperative Gleason scores were $(3+4)$ in 2 patient and $(3+3)$ in 6 patients. There was no positive surgical margin. Early postoperative complications were drain leakage $(n=1)$, atelectasis $(n=1)$, wound infection $(n=1)$ and fever $(n=1)$. Drain leakage was resolved during follow-up on the postoperative day 3 and the other complications were resolved with medical treatment. Postoperative continence was excellent. The first operated 6 patients were completely dry, whereas the last operated 2 patients, in their follow-up of $3^{\text {th }}$ and $4^{\text {th }}$ months, were using security pad. No other complications happened during the 4 to 12 months follow-up period. The serum level of PSA was less than $0.2 \mathrm{ng} / \mathrm{ml}$. The cosmetic result was excellent (Figure 4).

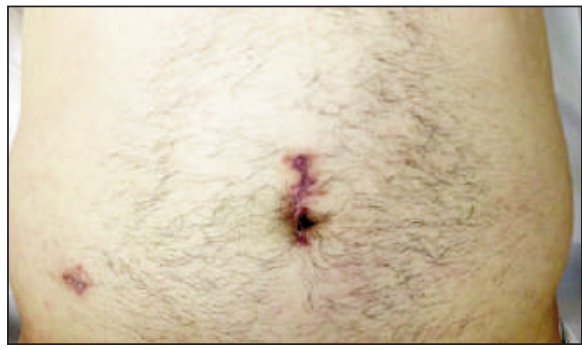

Figure 4.

Semilunar umbilical incision and

the cosmetic appearance.

\section{Discussion}

The treatment of localized prostate cancer has evolved over the last few decades from open surgery to laparoscopic, robotic and the newest entity single port robotic radical prostatectomy. An increasing number of centers worldwide have adopted LESS (8). However, it came with surgical limitations such as lack of triangulation, the instrument clashing, and the unfavorable ergonomics. The curved ports allow the instruments to make triangulation on targeted organ. Same-sided hand-eye control of the instruments is maintained by software of the da Vinci. It enables the surgeon control instrument on the right side of the screen with right hand and control instrument on the left side of the screen with left hand (Figure 5). The use of single-site instrumentation in urology was reported in several studies $(9,10)$. Increasing number of the series has resulted in experience with robot-assisted LESS radical prostatectomy (11). Currently literatures show that robot assisted laparoscopic prostatectomy is a safe and feasible procedure with favorable oncologic and functional outcomes $(2,7,12)$.

Anastomosis is the most challenging part of the SPORP. 


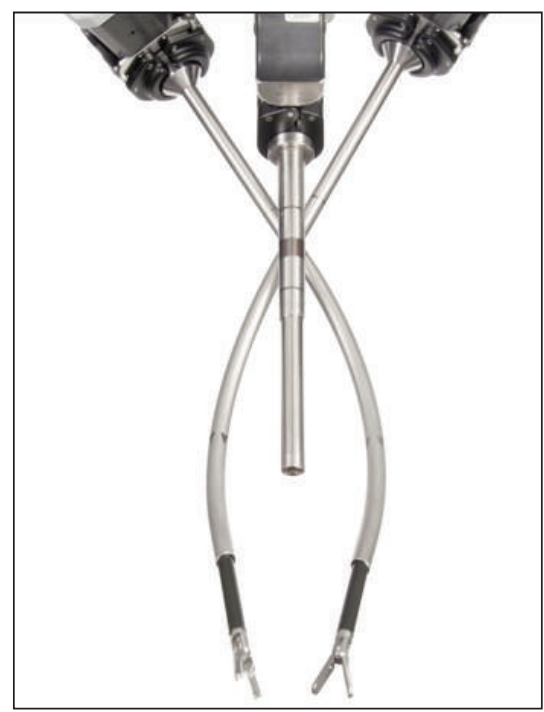

Figure 5. Surgeon control instrument.

Arms are not conventionally articulated like standard robotic arms.

Their range of motion is limited. In this point we prefer to use an extra $8 \mathrm{~mm}$ robotic port, which facilitates the suturing and is also used for drain extraction. Prostate excision and anastomosis time is even less than single port robotic radical prostatectomy (11). In the literature, additional port was used in $14.6 \%$ of cases (13). In addition to this, a $10 \mathrm{~mm}$ assistant port was inserted edge of single-site port without incising the fascia for the bedside assistant. This facilitates the challenges pointed in the literature related to bedside assisting during LESS (14-16). Conventional robotic procedure is comfortable for surgeon but port scars and complications due to number of ports like hernia or hemorrhage are main handicaps. In one study, mean blood loss during robotic radical prostatectomy was $135 \mathrm{cc}$, whereas in the present study mean blood loss was found to be $45 \mathrm{cc}$ (17). These handicaps led to identify the technique of SPORP. SPORP is more minimally invasive when compared with conventional multiple ports robotic or laparoscopic surgery $(18,19)$. Reducing the number of ports concluded with favorable outcomes, such as pain reduction and better cosmesis with robot-assisted LESS radical prostatectomy (20).

In an analysis of 1163 urologic LESS cases, the conversion rate was $4 \%$ to conventional laparoscopic/robotic surgery and $1.1 \%$ to open surgery (13). In the current study, all operations were completed successfully without conversion to standard robotic procedure or open surgery. Intraoperative complication rate was $3.3 \%$, whereas no intra operative complications occurred in our study (13).

The first operated 6 patients were completely dry, whereas the last operated 2 patients, in their follow-up of $3^{\text {th }}$ and $4^{\text {th }}$ months, were using security pad. Unfortunately with such a small sample the continence outcomes seem to be difficult to evaluate when compared with the literature (11).

As in our experience, minimally invasive techniques have demonstrated less blood loss and shorter convalescence (6). Making small incisions can limit the range of motion and visualization, but flexible robotic instruments have facilitated this difficulty. Choice of appropriate material such as Hem-o-lok clips for nerve sparing, barbed suture for anastomosis and long laparoscopic aspirator is also important.

In the current study, patients were relatively young (median age: $59.85 \mathrm{yrs}$ ) and in Tlc stage, had a low BMI (median: $27.6 \mathrm{~kg} / \mathrm{m}^{2}$ ), with a median prostate volume of $48 \mathrm{ml}$ and a median PSA of $6.91 \mathrm{ng} / \mathrm{ml}$. Our study population is close to the literature on LESS robotic prostatectomy (12). Surgical margin results seem better than single port laparoscopic radical prostatectomy $(11,21)$. The most difficult part of the procedure is that it requires robotic and laparoscopic experience.

\section{Conclusions}

In conclusion, SPORP does not seem as difficult as presented in the initial reports. It is relatively easy for urologists who are skilled in traditional laparoscopic and robotic surgeries to master SPORP. In addition the procedure would be easier if the arms were articulated. Trifecta is possible with pain reduction and excellent cosmetic results. However, the long-term outcomes of this surgery need further investigations.

\section{REFERENCES}

1. Jung JH, Kim HW, Oh CK, et al. Simultaneous robot assisted laparoendoscopic single site partial nephrectomy and Standard radical prostatectomy. Yonsei Med J. 2014; 55:535-8.

2. Boncher N, Vricella G, Greene G, Madi R. Concurrent robotic renal and prostatic surgery: initial case series and safety data of a new surgical technique. J Endourol. 2010; 24:1625-9.

3. Jung JH, Arkoncel FR, Lee JW, et al. Initial clinical experience of simultaneous robot assisted bilateral partial nephrectomy and radical prostatectomy. Yonsei Med J. 2012; 53:236-9.

4. Cáceres F, Cabrera PM, García-Tello A, et al. Safety study of umbilical single-port laparoscopic radical prostatectomy with a new DuoRotate system. Eur Urol. 2012; 62:1143-9.

5. Kaouk JH, Goel RK, Haber GP, et al. Single-port laparoscopic radical prostatectomy. Urology. 2008; 72:1190-3.

6. Parsons JK, Bennett JL. Outcomes of retropubic, laparoscopic, and robotic-assisted prostatectomy. Urology. 2008; 72:412-6.

7. Rha KH. Robot-assisted laparascopic radical prostatectomy. Korean J Urol. 2009; 50:97-104.

8. Kaouk JH, Autorino R, Kim FJ, et al. Laparoendoscopic single-site surgery in urology: worldwide multi-institutional analysis of 1076 cases. Eur Urol. 2011; 60:998-1005.

9. Haber GP, White MA, Autorino R, et al. Novel robotic da Vinci instruments for laparoendoscopic single-site surgery. Urology. 2010; 76:1279-82.

10. Cestari A, Buffi NM, Lista G, et al. Feasibility and preliminary clinical outcomes of robotic laparoendoscopic single-site (R-LESS) pyeloplasty using a new single-port platform. Eur Urol. 2012; 62:175-9.

11. White MA, Haber GP, Autorino R, et al. Robotic laparoendoscopic single-site radical prostatectomy: technique and early outcomes. Eur Urol. 2010; 58:544-50.

12. Kaouk JH, Haber GP, Autorino R, et al. A Novel Robotic System for Single-port Urologic Surgery: First Clinical Investigation. Eur Urol. 2014; 66:1033-1043. 
13. Autorino R, Kaouk JH, Yakoubi R, et al. Urological laparoendoscopic single site surgery: multi-institutional analysis of risk factors for conversion and postoperative complications. J Urol. 2012; 187:1989-94.

14. White MA, Haber GP, Autorino R, et al. Robotic laparoendoscopic single-site surgery. BJU Int. 2010; 106:923-7.

15. White MA, Autorino R, Spana G, et al. Robotic laparoendoscopic single site urological surgery: analysis of 50 consecutive cases. J Urol. 2012; 187:1696-701.

16. Autorino R, Kaouk JH, Stolzenburg JU, et al. Current status and future directions of robotic single-site surgery: a systematic review. Eur Urol. 2013; 63:266-80.

17. Tasci AI, Bitkin A, Ilbey YO, et al. Robot-assisted laparoscopic radical prostatectomy: initial experience with first 112 cases. J Robot Surg. 2012; 6:283-8.

18. Clayman RV, Box GN, Abraham JB, et al. Rapid communication: transvaginal single-port NOTES nephrectomy: initial laboratory experience. J Endourol. 2007; 21:640-4.

19. Desai MM, Berger AK, Brandina R, et al. Laparoendoscopic single-site surgery: initial hundred patients. Urology. 2009; 74:805 12.

20. Autorino R, Cadeddu JA, Desai MM, et al. Laparoendoscopic single site and natural orifice transluminal endoscopic surgery in urology: a critical analysis of the literature. Eur Urol. 2011; 59:26-45.

21. White WM, Haber GP, Goel RK, et al. Single-port urological surgery: single-center experience with the first 100 cases. Urology. 2009; 74:801-4.

\section{Correspondence}

Volkan Tugcu, MD

Abdulmuttalip Simsek, MD (Corresponding Author)

simsek76@yahoo.com

Ismail Evren, MD

Kamil Gokhan Seker, MD

Ramazan Kocakaya, MD

Bugra Dogukan Torer, MD

Arda Atar, MD

Ali Ihsan Tasci, MD

Department of Urology, Bakirkoy Dr.Sadi Konuk Education

and Research Hospital

Tevfik Saglam Caddesi No:11 Zuhuratbaba/Bakirkoy, Istanbul 34147, Turkey 